\title{
Exploring Views for Goal-oriented Requirements Comprehension
}

Lyrene Silva1', Ana Moreira², João Araújo², Catarina Gralha², Miguel Goulão² and Vasco Amaral²

1 Universidade Federal do Rio Grande do Norte (Brazil),

2Universidade NOVA de Lisboa (Portugal) 


\section{There is a diversity of options in the software development process}

need to use
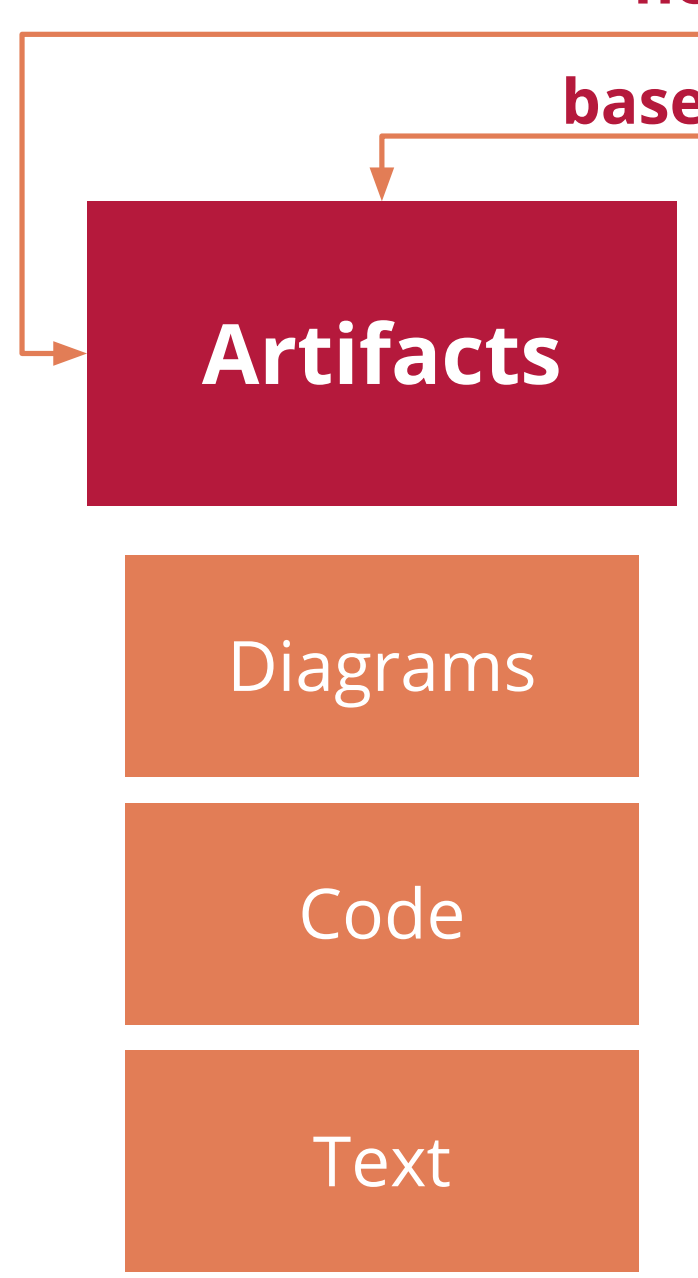


\section{But what is the problem?}

Requirements artifacts are often too large or too complex to be quickly understood or queried by different stakeholders

Multiple views are broadly employed in requirements modeling for very specific purposes

These views do not offer interactive features to allow stakeholders browsing the information according to their needs

Views of the requirements should be configured to their particular needs 


\section{Requirements exploration}

It is a process to navigate through requirements artifacts, aiming at comprehending their structure and content

Reasons to provide mechanisms for requirements exploration:

1) Requirements artifacts are often used by people who have not created them

2) Stakeholders need to search information on these artifacts

3) Exploration mechanisms can help navigating through the entire documentation to find the elements associated with a specific point of interest 


\section{Viewpoint, point-of-view, view and visualization}

\section{Viewpoint and point-of-view}

opinion that a person has about a given subject

\section{View}

includes a representation language

\section{Visualization}

includes a representation mechanism such as zoom, filter, details on demand, history log, overview, ... 


\section{Proposing 3 views for requirements exploration}

\section{Big Picture view}

Generates an overview for a source model

Offers the ability to expand and reduce the details on demand

Organizes the model information on levels of importance or by aggregation 


\section{Proposing 3 views for requirements exploration}

\section{Big Picture view}

Generates an overview for a source model

Offers the ability to expand and reduce the details on demand

Organizes the model information on levels of importance or by aggregation

\section{Syntax-based view}

Filters the types of language elements that will be visualized 


\section{Proposing 3 views for requirements exploration}

\section{Big Picture view}

Generates an overview for a source model

Offers the ability to expand and reduce the details on demand

Organizes the model information on levels of importance or by aggregation

\section{Syntax-based view}

Filters the types of language elements that will be visualized

\section{Concern-based view}

Filters concerns through meta-data, system lexicon (key words) or semantic similarity 


\section{Conceptual model: exploratory views of requirements}

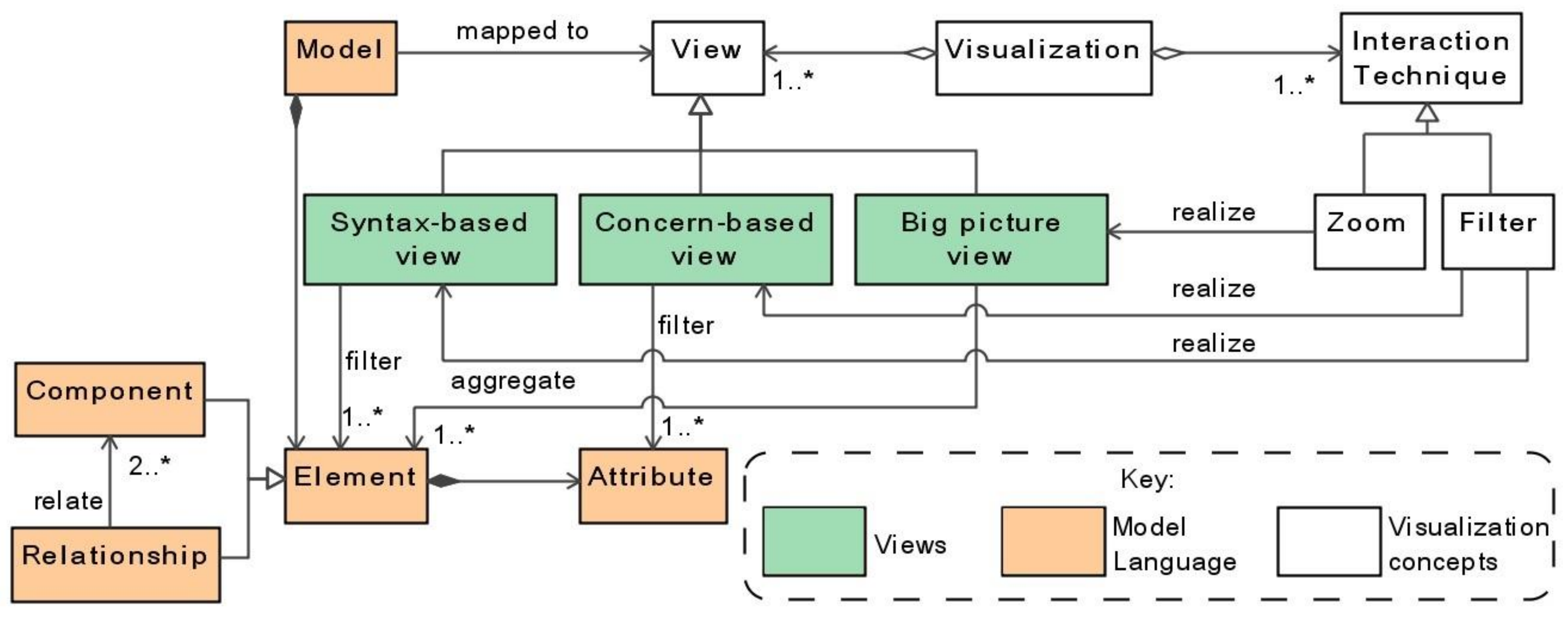




\section{Demonstration: Health Care System}
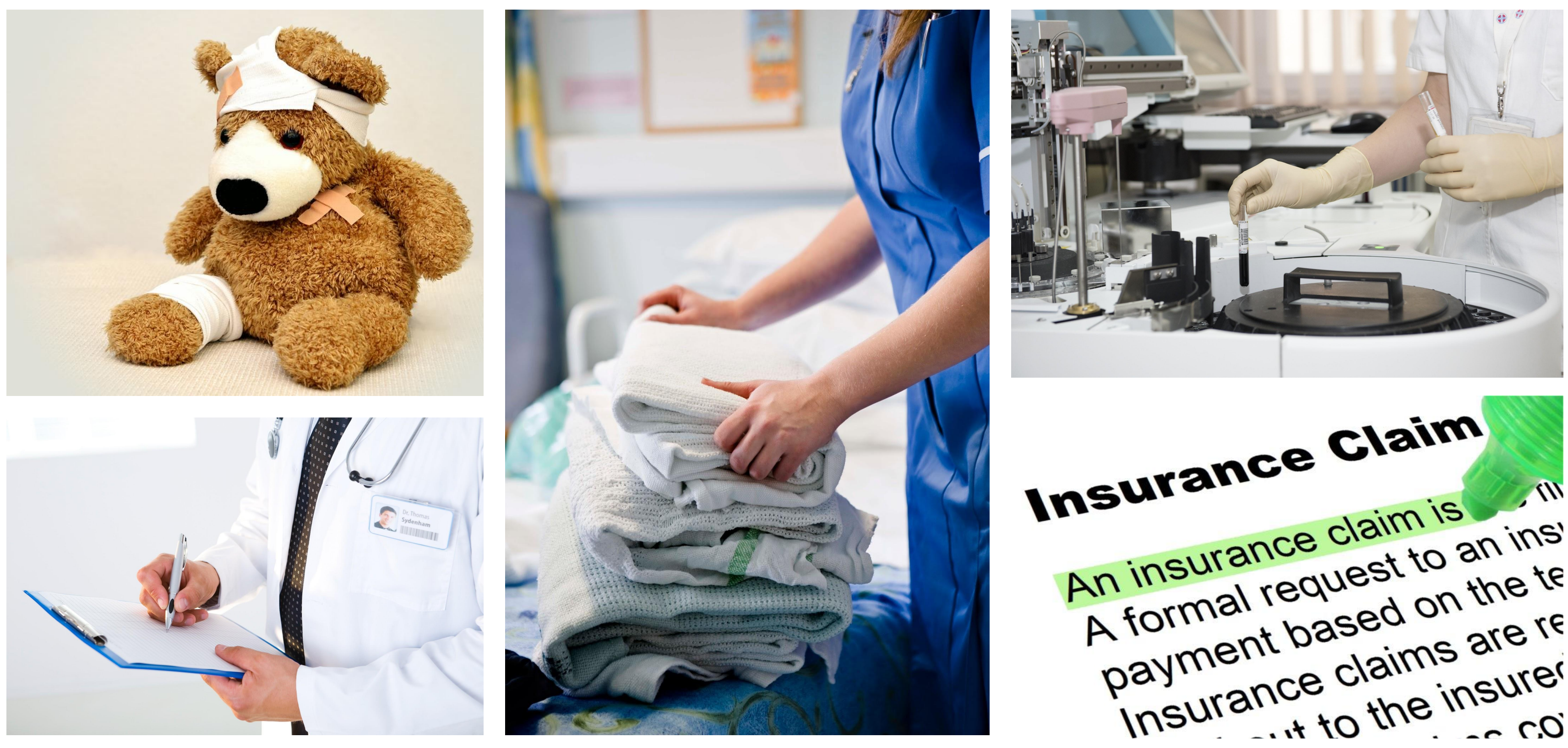

Insurance Claim An insurance claim to an ins A formal rased on the $r e$ paymente claims are insuran to the insure 


\section{Demonstration: $i *$ model for Health Care}

\section{Numbers for Health Care System (HCS):}

13 actors

13 goals

41 tasks

\section{5 links}

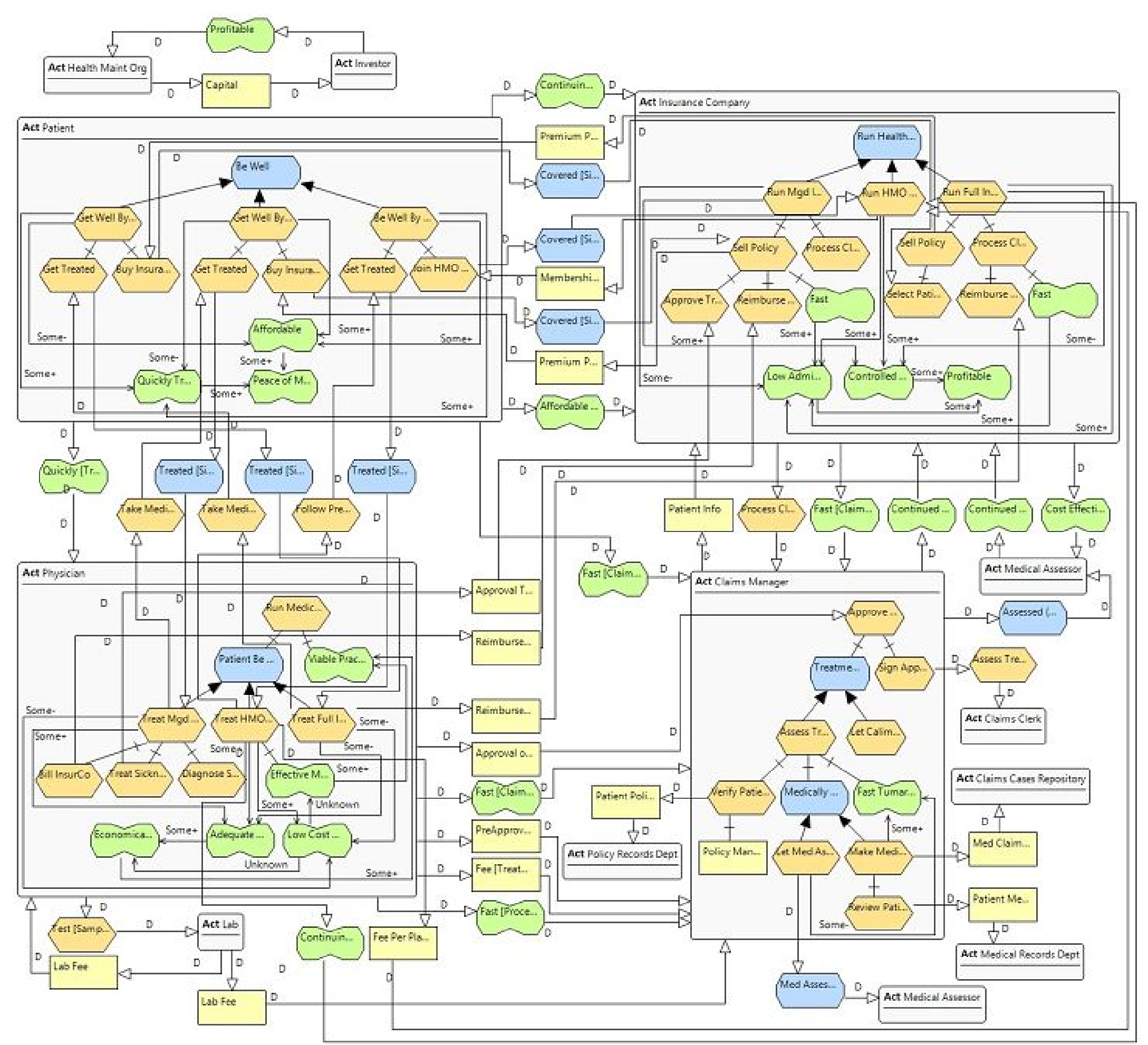




\section{Big Picture view expanded}

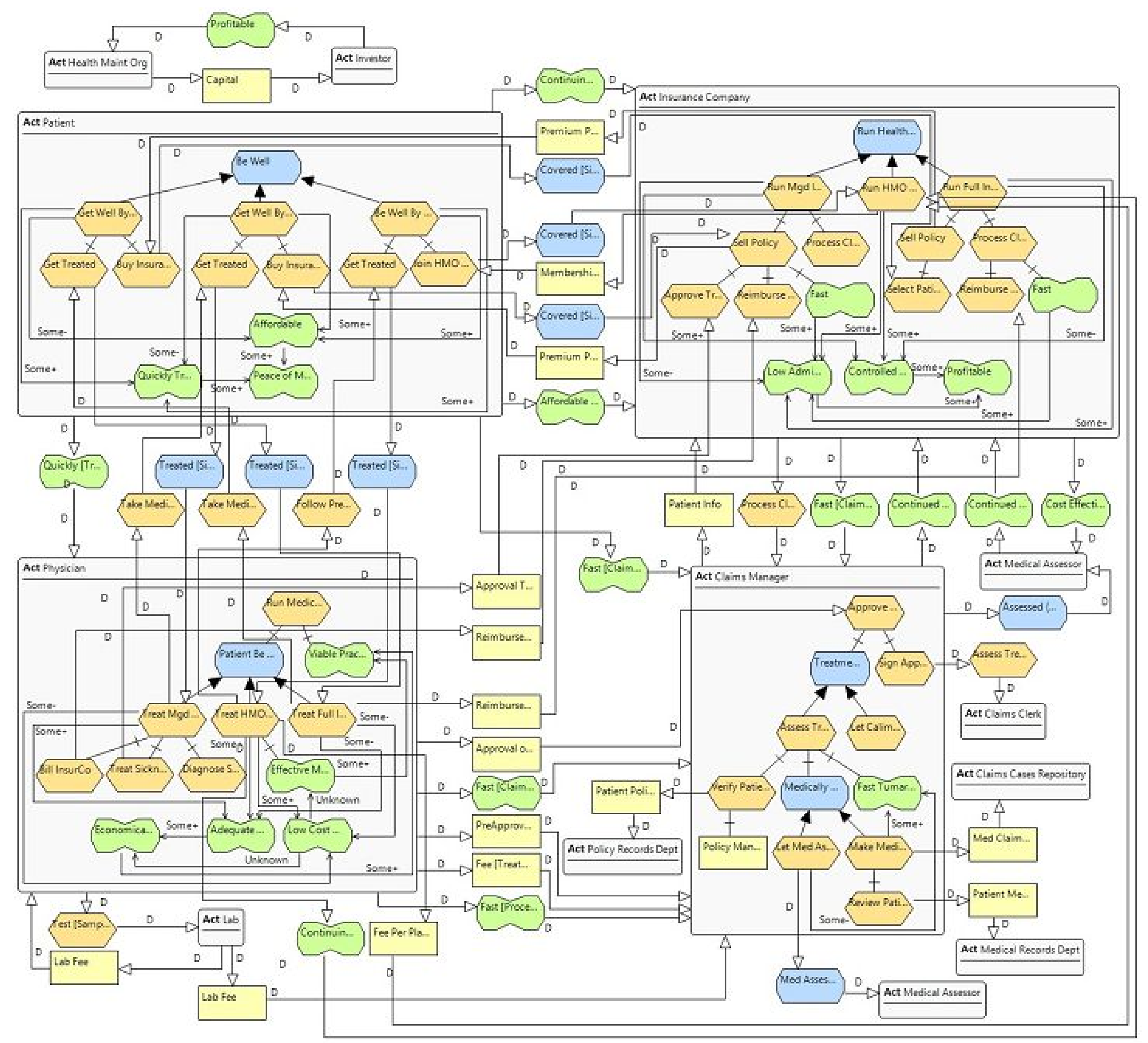




\section{Big Picture view with both actors and dependencies collapsed}

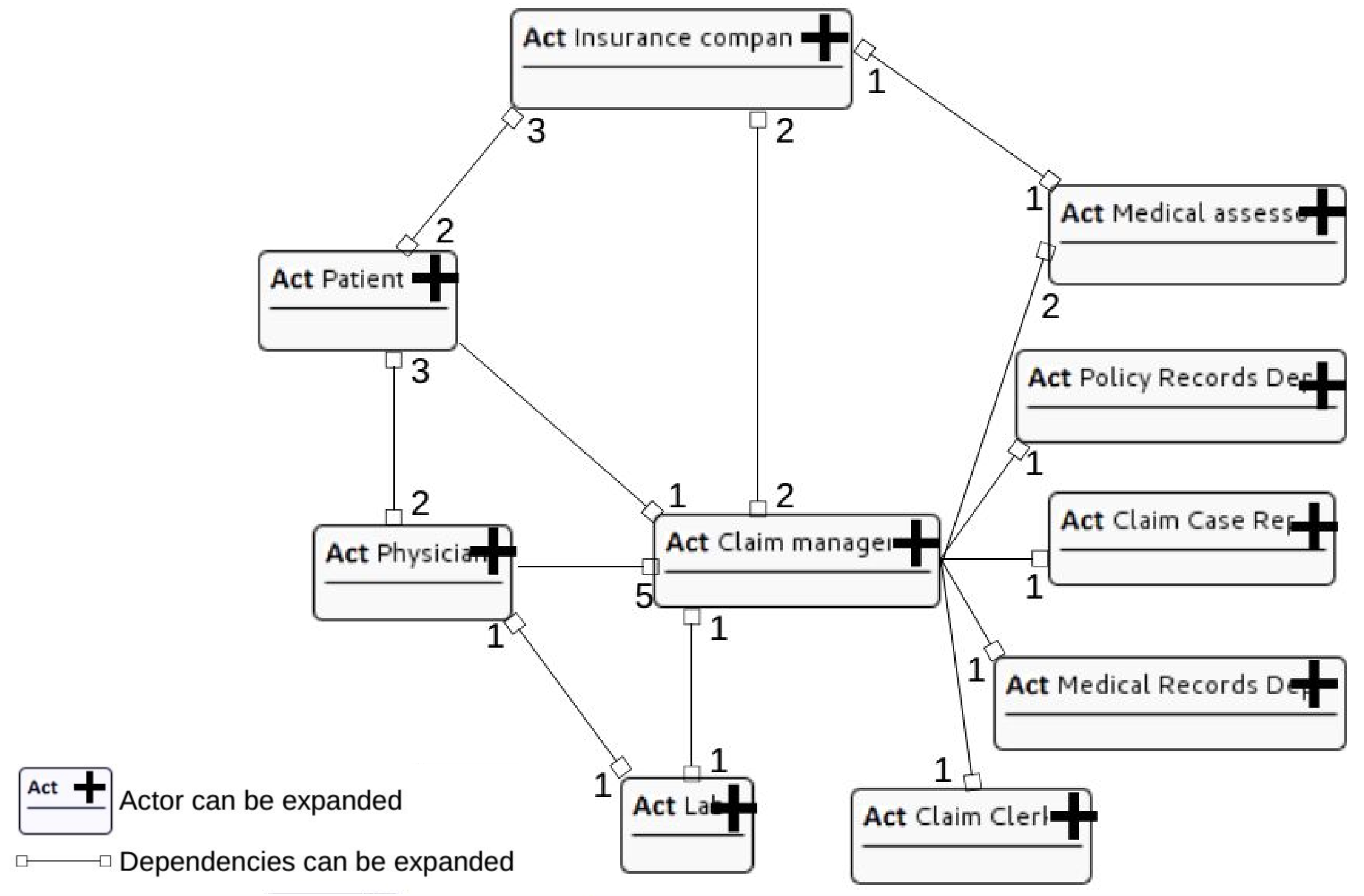




\section{Big Picture view with both actors and dependencies collapsed}

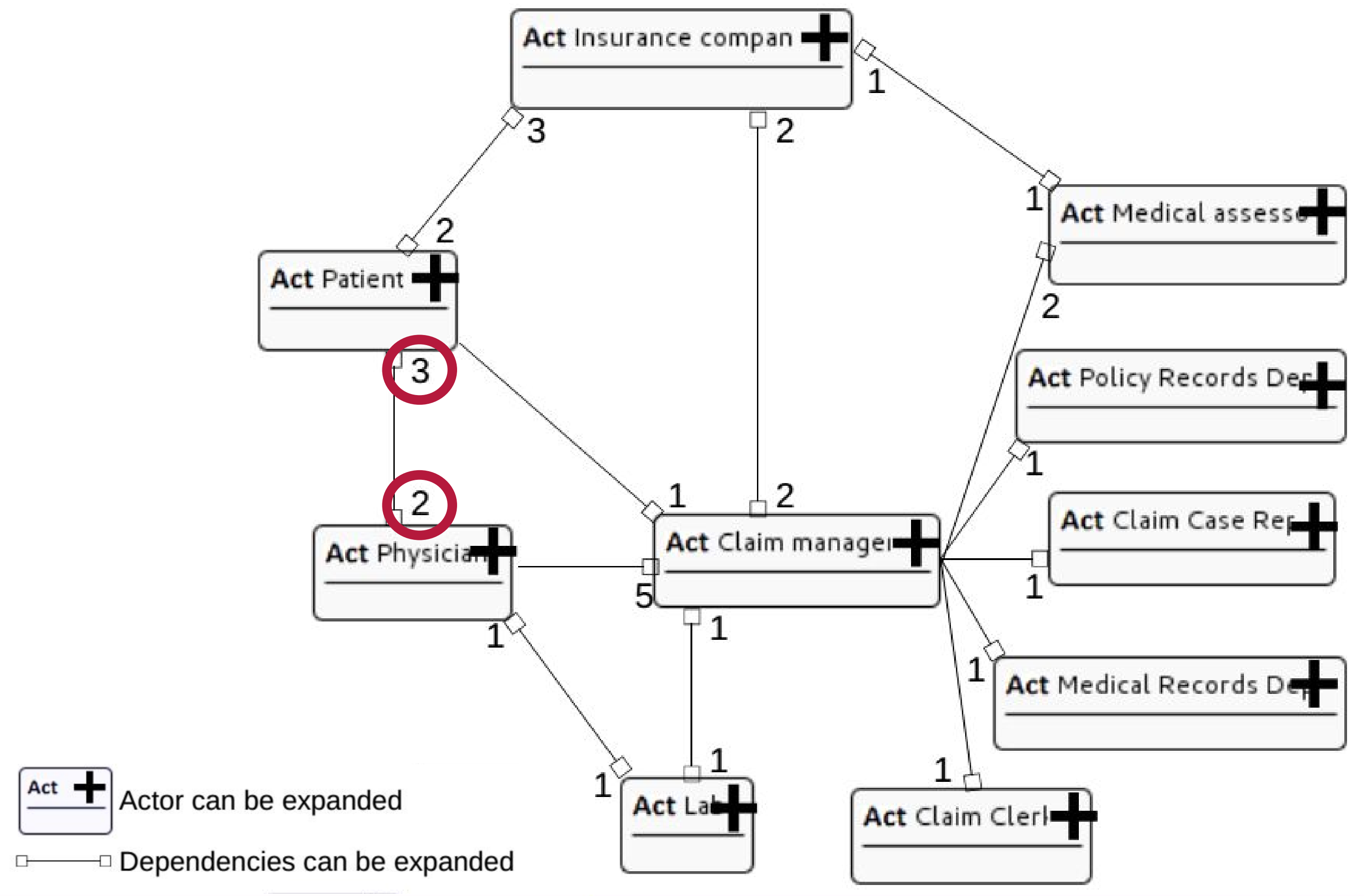




\section{Big Picture view with an expanded actor and link}

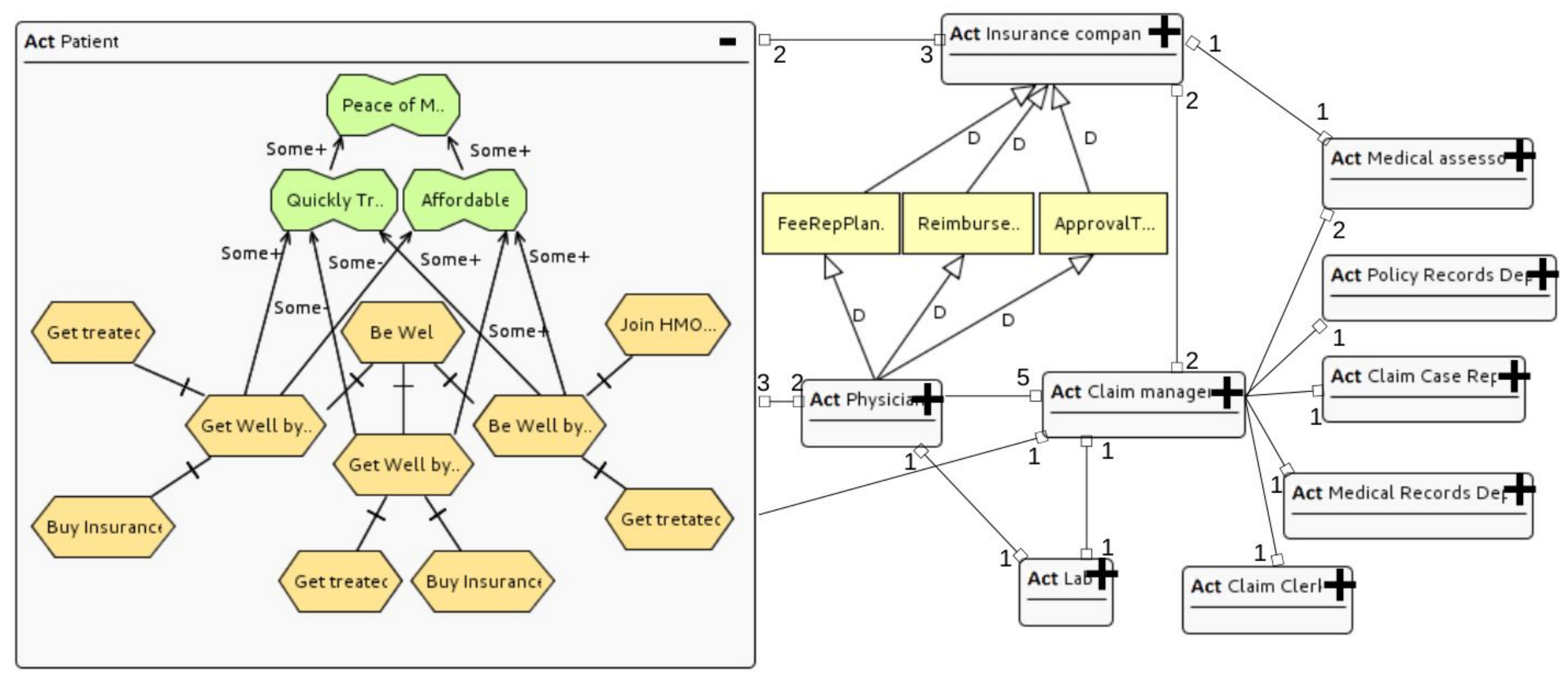




\section{Syntax-based view: filtering resource dependencies}

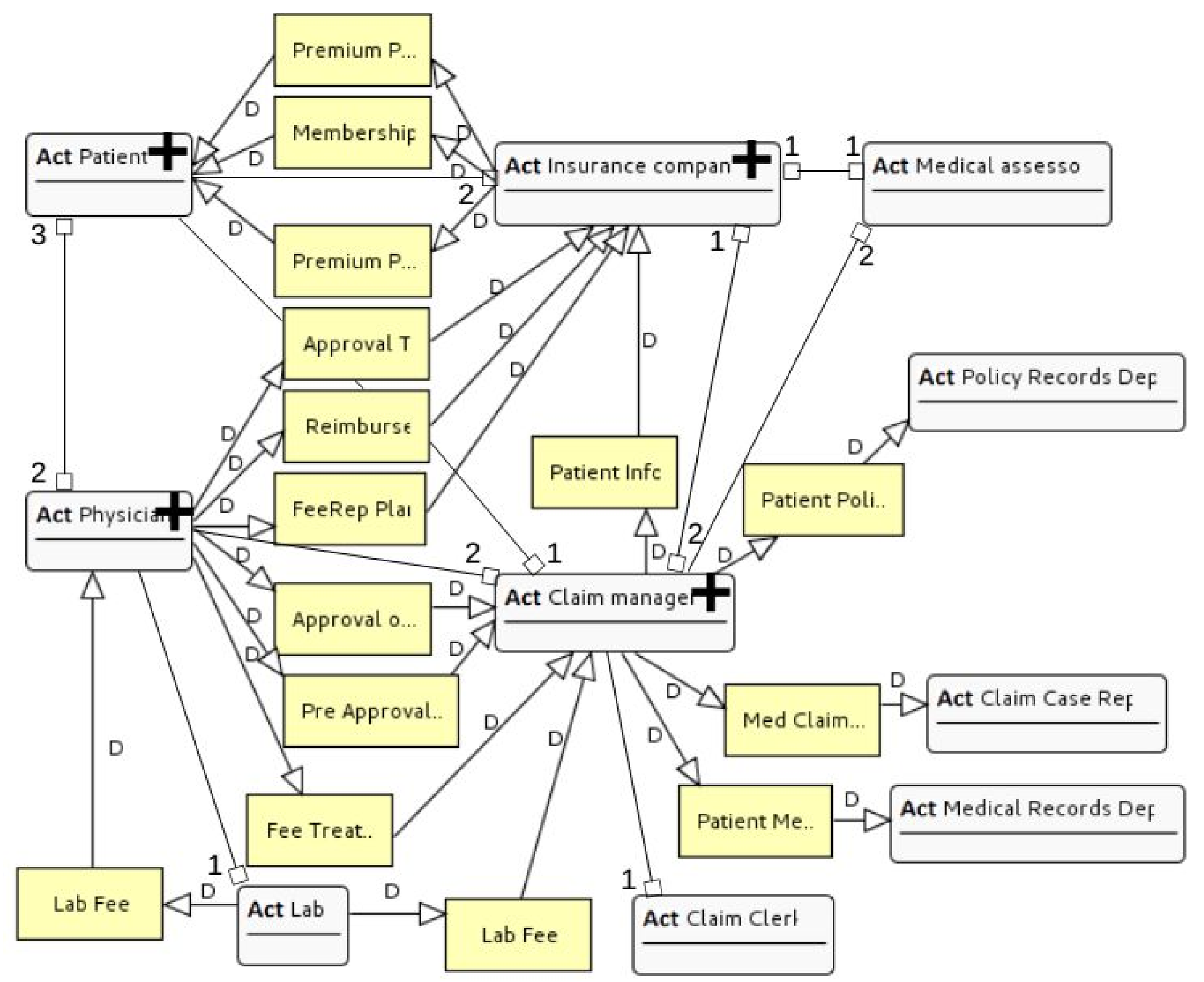




\section{Concern-based view: filtering the string Cost, considering a distance of one}
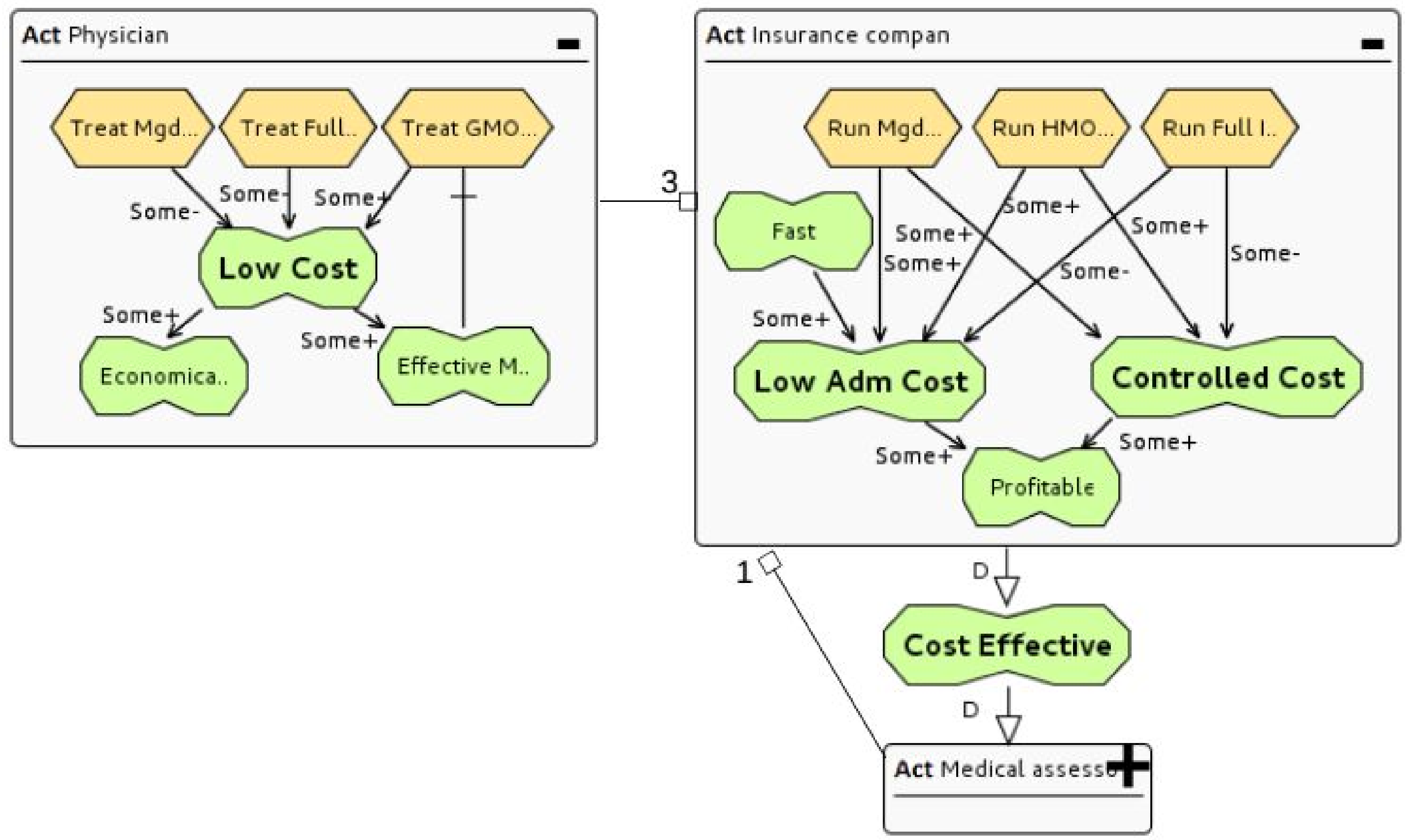


\section{Tool support for the syntax-based view}

(d) default.istar_diagram £

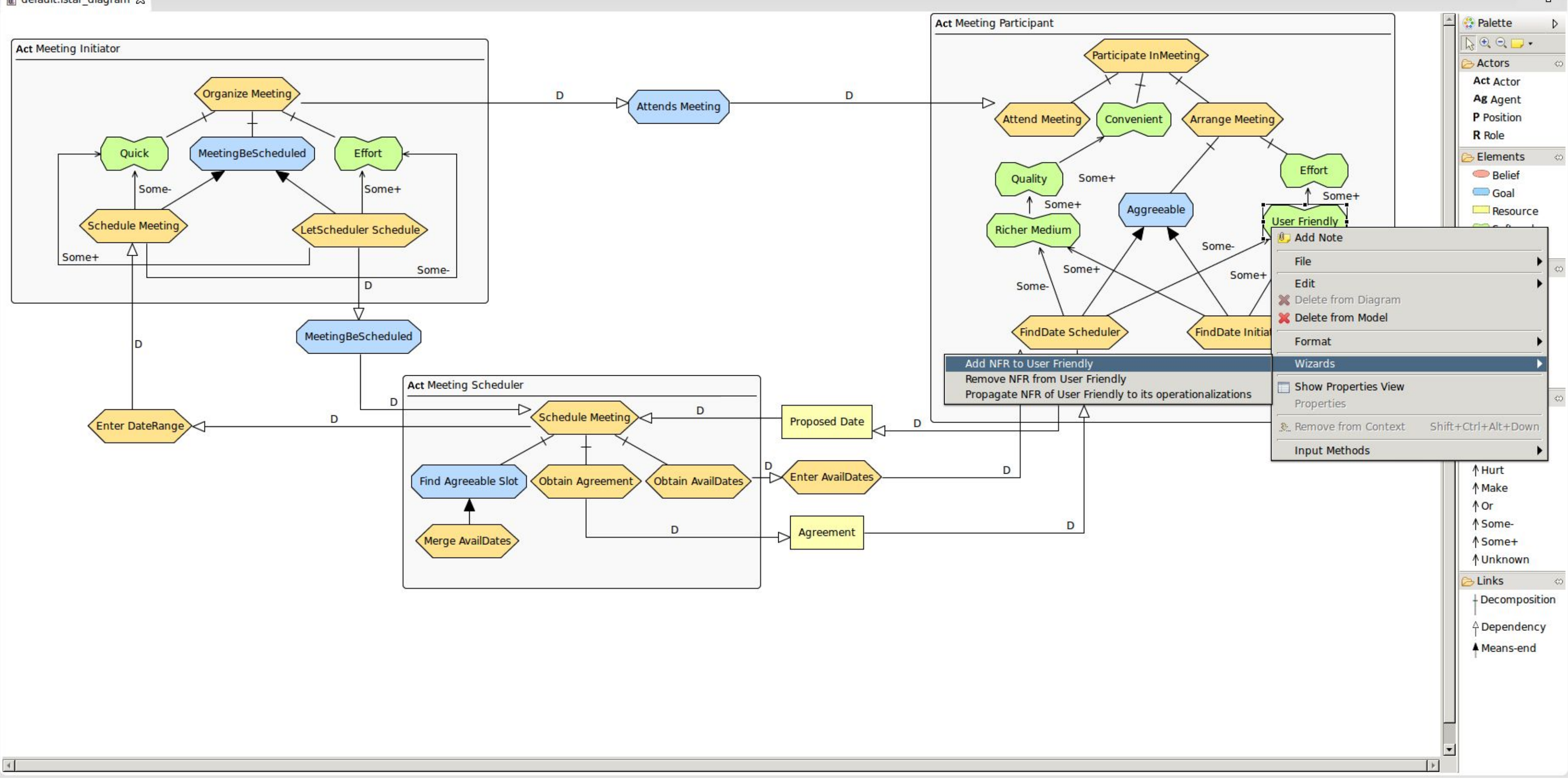




\section{Discussion and current challenges for using multiple view for requirements exploration}

In the requirements engineering process, many models are generated. It is necessary to provide exploration mechanisms to navigate through them, instead of only navigating an isolated model

The tools implementing this complementary views should provide mechanisms to allow users to interact directly with the visual elements

Users may interact with the source model as well as with the views. It is necessary to generate a view about the path followed to the achieved result

Interaction mechanisms include aspects from human-computer interaction have not been taken into consideration yet 


\section{Summary and conclusions}

We proposed 3 views for requirements exploration:

big picture, syntax-based and concern-based

They are based on the interaction tasks zoom and filter

They capture 3 manners of abstracting a model, by:
1) decreasing it's amount of elements
2) allowing stakeholders to search
3) focusing on information of interest

Can be applied to other kinds of models: we have done it for use cases

Our views deal with the complexity of requirements models

Without this kind of mechanisms, more stakeholders effort is demanded to find and analise relevant information in the system model 


\section{Future work}

Investigate how tools can be prepared for supporting our views

Define a process to instantiate our views to other requirement models

Conduct experimental evaluations of the impact of introducing the proposed views in requirements tools

Evaluate how the proposed views impact on the efficiency and effectiveness of different stakeholders while performing requirements exploration 


\section{Thank you}

\section{Questions?}

